LBL --29192

DE9 I 004245

\title{
Electronic Properties of Hydrogen-Related Complexes in Pure Semiconductors
}

\author{
Eugene E. HALLER
}

\author{
University of California at Berkeley \\ and \\ Materials and Chemical Sciences Division \\ Lawrence Berkeley Laboratory \\ 1 Cyciotron Road \\ Berkeley, Califomia 94720 USA
}

This work was supported by the Director, Office of Energy Research, Office of Basic Energy Scinces, Materials Science Division of the U.S. Department of Energy under Contract No. DE-AC.03-76SF00098. 


\title{
Electronic Properties of Hydrogen-Related Complexes in Pure Semiconductors
}

\author{
Eugene E. HALLER \\ University of California at Berkeley \\ and \\ Lawrence Berkeley Laboratory \\ Berkeley, California 94720 USA
}

\begin{abstract}
Hydrogen has been shown to activate the neutral impurities carbon, silicon and oxygen in ultra-pure germanium and form shallow level complexes. The double acceptors beryllium and zinc in silicon and germanium, as well as the triple acceptor copper in germanium, can be partially passivated, leading to single hole acceptors. The study of the electronic level spectrum of the single carrier bound to these centers at low temperatures has provided much information on symmetry and composition. Most centers reveal a symmetry axi; along [111] and are static. In some cases hydrogen has been found to tunnel between equivalent real space positions. Photothermal Ionization Spectroscopy (PTIS) has been the most important tool for the study of the optical transitions of the hole (electron) in these hydrogen containing complexes. This photoconductivity technique combines high sensitivity with high resolution and permits the study of shallow acceptors or donors present at concentrations as low as $10^{8} \mathrm{~cm}^{-3}$. Even lower limits may be attained under favorable circumstances.
\end{abstract}




\section{Introduction}

Early studies of the diffusion and the solubility of hydrogen in silicon [1] and germanium $[1,2]$ in the late 1950's showed that this smallest of all elements is a fast, interstitially moving impurity with a low solubility that appeared technologically unimportant. More than twenty years later, during the development of ultra-pure germanium single crystals for large volume gamma radiation detectors $[3,4]$, hydrogen was found to combine with the isoelectronic neutral impurities carbon and silicon and with neutral oxygen to form the shallow acceptors $A(\mathrm{H}, \mathrm{C})$ and $A(\mathrm{H}, \mathrm{Si})$ and a shallow donor $\mathrm{D}(\mathrm{H}, \mathrm{O})$, respectively [5]. This discovery, which was significantly facilitated by high resolution far inirared PTIS, led to a renewed interest in the interactions of hydrogen in crystalline semiconductors. Sah et al.'s [6] discovery of shallow acceptor passivation in MOS structures followed by the RF plasma hydrogenation experiments of Pankove et al. $[7,8]$ in silicon led to the worldwide research activities which have constituted an important part of semiconductor research for the past seven years.

This review focuses on the early studies of $A(\mathrm{H}, \mathrm{C}), A(\mathrm{H}, \mathrm{Si})$ and $\mathrm{D}(\mathrm{H}, \mathrm{O})$, on the partially hydrogen passivated multivalent acceptors, and on PTIS, the single most important tool for the study of electrically active centers present at low concentrations.

\section{Experimental Techniques}

\subsection{Crystal growth}

The development of ultra-pure germanium single crystals for gar....2 ray detector applications required special materials for the crystal growth process and highly sensitive analytical techniques. A net-dupant concentration $\left|N_{A}-N_{D}\right| \cong 10^{10} \mathrm{~cm}^{-3}$ was required in order to obtain the necessary depletion layer thicknesses of $\geq 1 \mathrm{~cm}$ at acceptable bias values $(\leq 5000 \mathrm{~V})$. Hall [9] discussed the feasibility of obtaining such crystals as early as

1966. Using the Czochralski melt growth technique [10] one had to select a crucible 
material and an ambient which would lead to the extremely low residual impurity concentrations. Synthetic silica (vitreous quartz) and palladium purified hydrogen offered the best prospects. Today's industrial processes still use synthetic silica and $\mathrm{H}_{2}$ and produce $7 \mathrm{~cm}$ diameter single crystals of ultra-pure germanium.

The slow reduction of silica by the germanium melt leads to free silicon, an isoelectronic impurity, and oxygen, a neutral impurity when isolated. A state-of-the-art single crystal contains between $10^{13}-10^{14} \mathrm{~cm}^{-3}$ of each of these impurities. Growth experiments in tritium containing $\mathrm{H}_{2}$ atmospheres [11] were used to determine the total hydrogen concentration which lies between 1 to $3 \times 10^{14} \mathrm{~cm}^{-3}$ in 1 atm $\mathrm{H}_{2}$ grown crystals. In crystals grown from a graphite crucible one finds a carbon concentration $\cong 10^{14} \mathrm{~cm}^{-3}$ [12]. In addition to these electrically neutral impurities one typically finds the shallow level impurities aluminum and phosphorus, and in special cases gallium and indium at concentrations $\leq 10^{10} \mathrm{cis}^{-3}$. Thus the designation of these materials as "ultra-pure" refers only to the electrically active residual impurities.

\subsection{Hall effect and Photothermal Ionization Spectroscopy}

Quantitative analysis of such small concentrations of shallow donors and acceptors can be achieved with electrical measurements. Variable temperature Hall effect measurements $[13,14]$ give the type of conduction, accurate net-concentrations $\left|N_{A}-N_{D}\right|$ and energy level values to within 5-10\%. Free carrier freeze-out curves do not allow differentiation between the various shallow level impurities, all of which have ground state energies close to $10 \mathrm{meV}$.

The close proximity of the vari us shallow level energies in Ge requires a high resolution spectroscopic technique to distinguish between the various acceptor and donor species. 
PTIS has turned out to be one of the most appropriate tools for this purposc. Because of its importance we will describe this powerful spectroscopic technique in some detail.

Fig. 1 shows the two-step process which characterizes PTIS. The optical excitation of a donor or acceptor bound carrier from the ground state to a bound excited state is followed by thermal excitation into the band. The change in free carrier concentration leads to a change in electrical conductivity of the sample which can be measured with great sensitivity. Lock-in techniques allow narrow band measurements and lead to excellent signal to noise ratios. PTIS has an optimum temperature range for a given energy level. The temperature must be sufficiently low so that the majority of the donors or acceptors are frozen-out, i.e., neutral. On the other hand it must be high enough so that phonons of sufficient energy can transport the optically excited carrier from the excited state to the band continuum. Fig. 2 shows schematically the evolution of a PTIS spectrum with temperature.

A key condition for successful PTIS is the formation of good electrical contacts on the sample under study. A number of techniques have been developed to form low noise, ohmic contacts which function at the low temperatures required. Ion implantation followed by thermal annealing at moderate temperature, diffusion, or simple vacuum evaporation or soldering of metals forming relatively shallow energy barriers have been used successfully. The choice of the particular contact formation technique depends strongly on the sample to be studied. If a sample can be easily contaminated at the elevated temperatures required for contact formation one has to resort to low temperature techniques. Copper, for example, has a significant solubility and a high diffusion coefficient in germanium. If kept at temperatures below $\sim 350^{\circ} \mathrm{C}$ copper contamination can be avoided. This temperature limit is sufficiencly high to allow thermal activation of boron or phosphorus implanted at low 
energies $(<50 \mathrm{keV})$ in germanium. Rapid thermal annealing (RTA) offers efficient reduction of impurity contamination during contact formation.

PTIS was discovered by Lifshits and Ya'Nad [15]. Using Fourier transform spectroscopy [16], full use can be made of the high resolution potential of PTIS with ultra-pure semiconductors. In pure semiconductors with a small concentration of structural defects, the lifetime of a carrier in a bound excited state can be very long, on the order of $100 \mathrm{~ns}$ [17]. The individual impurities are well isolated from each other and wave function overlap is extremely small even for highly excited states. The concentration of compensated ionized acceptors and donors lies in the $10^{10} \mathrm{~cm}^{-3}$ range, too small to create electric fields which could cause measureable Stark effects. Haller et al. [18] and Navarro et al. [19] have studied the width of optical $1 s-n p$ transitions and the lifetime of excited states of the $\mathrm{D}(\mathrm{H}, \mathrm{O})$ donor in ultra-pure germanium. They found that the excited state lifetime approaches the fundamental limit given by final state mixing $[20,21]$.

In an interesting modification of standard PTIS, bandedge light ( $\left.h v \cong E_{g a p}\right)$ is used to generate free holes and electrons ir the sample under study. In this case the compensated minority impurities, which are typically ionized at any temperature and escape spectroscopic observation, can capture free carriers and become spectroscopically active. Using $p$-type ultra-pure germanium Skolnick et al. [22] performed shallow acceptor PTIS, as shown in Fig. 3a. Adding band edge light neutralizes shallow donors which become accessible to PTIS. In the case of $p$-type ultra-pure germanium, the free electrons originating from donor PTIS will recombine efficiently with majority carriers, holes, reducing their concentration and leading to a reduction in conductivity. This in turn leads to negative peaks in the PTIS spectrum (Fig. 3b). In the absence of efficient recombination between majority and minority carriers all peaks will be of the same polarity. Darken and Hyder [23] as well as Van de Steeg et al. [24] have given a quantitative analysis of PTIS in 
Ge. Van de Steeg et al. [25] proposed a search for fractionally charged impurities using PTiS. They arrived at a sensitivity limit of $10^{5} \mathrm{~cm}^{-3}$ fractionally charged impurities. This is an impressive illustration of the potential of PTIS.

In a recent PTIS study of shallow donors in pure and closely compensated GaAs epilayers, Wolk et al. [26] have shown that the neutralization of majority and minority dopants through band edge light leads to a large reduction of Stark broadening produced by random Coulomb fields of the various positive (donors) and negative (acceptors) ionized dopants. This resulted in the sharpest donor lines ever recorded in GaAs and led to the first experimental verification of the splitting of the $(2 n+1) p$-states as was theoretically predicted by Larsen [27].

PTIS has been very effective for the study of hydrogenic impurities and of deep levels in $\mathrm{Si}$ with effective mass theory (EMT) like bound excited states. Chalcogen double donors [28] and a number of transition and noble metal impurities [29] have been investigated. In many of these studies a process different from PTIS has been very helpful. If a sample contains both shallow and deep levels, it is quite likely that the photoconductive continuum of the shallow impurity(ies) reaches well into and beyond the photon energy range in which characteristic deep level transitions occur. These transitions remove photons whic would have contributed to the shallow level continuum, thereby leading to negative absorption lines. This self-absorption process is not a PTIS process. It is equivalent to the merging of a silicon photoconductive detector and a silicon sample doped with deep level impurities into one sample. The advantages gained from this self- or internal absorption have been amply demonstrated in numerous Si studies by the Lund group [30]. 


\section{Electrically Active Hydrogen-Containing Complexes}

\subsection{The static centers $A(\mathrm{H}, \mathrm{Si}), A(\mathrm{H}, \mathrm{C}), A(\mathrm{Be}, \mathrm{H})$ and $A(\mathrm{Zn}, \mathrm{H})$ in $\mathrm{Ge}$}

The four hydrogen containing complexes $A(\mathrm{H}, \mathrm{Si}), A(\mathrm{H}, \mathrm{C}), A(\mathrm{Be}, \mathrm{H})$ and $A(\mathrm{Zn}, \mathrm{H})$ are static trigonal centers. The first two were discovered in germanium in crystals grown from silica and graphite crucibles while the latter two appear in crystals intentionally doped with $\mathrm{Be}$ and $\mathrm{Zn}$.

$A(\mathrm{H}, \mathrm{Si})$ can be generated by rapidly quenching a small sample of hydrogen atmosphere grown ultra-pure germanium from around $425^{\circ} \mathrm{C}$ [3]. A small fraction $(\sim 0.1 \%)$ of the hydrogen must be present in a mobile, interstitial form at that temperature. During the quenching process the thermal energy of $\mathrm{H}$ rapidly diminishes and it becomes trapped by the nearest Si impurity. Theory predicts a binding energy of a few tens of meV which is compatible with the observation that $A(\mathrm{H}, \mathrm{Si})$ is not stable at room temperature. Substitution of $\mathrm{H}$ with $\mathrm{D}$ leads to an isotope shift of $21 \mu \mathrm{eV}$ in the ground state of $A(\mathrm{H}, \mathrm{Si})$ [31].

The acceptor $A(\mathrm{H}, \mathrm{C})$ is found in Ge crystals grown from a melt contained in a graphite crucible. The complexes are present in as grown crystals and stable up to $\sim 200^{\circ} \mathrm{C}$. No isotope shift has been observed.

$A(\mathrm{H}, \mathrm{Si})$ and $A(\mathrm{H}, \mathrm{C})$ were the first shallow acceptors which exhibited a split $l \mathrm{~s}$-state while the np-states are perfectly EMT like; i.e., "hydrogenic" in nature. The wavefunctions of shallow acceptors are combinations of the wavefunctions describing the top of the $p 3 / 2$ valence band. This valence band is fourfold degenerate and leads to $l \mathrm{~s}$ states with $\Gamma_{8}$ symmetry ( $3 / 2$ spin holes). The observed splitting must be caused by additional effects acting on the $1 \mathrm{~s}$ state but not on the $n \mathrm{p}$ states. Kahn et al. [32] proposed an internal stress 
model which quantitatively describes the splitting of the $1 \mathrm{~s}$-state and the behavior of the $1 \mathrm{~s}$ states of $A(\mathrm{H}, \mathrm{Si})$ and $A(\mathrm{H}, \mathrm{C})$ under externally applied uniaxial stress in all major crystal orientations. The very small isotope shift in the ground state of $A(\mathrm{H}, \mathrm{Si})$ cannot be explained with this model and is presumably due to the difference in zero-point motion of hydrogen and of deuterium. The np.states are not affected by the internal stress because their wavefunctions vanish at the impurity core.

There are a number of unanswered questions regarding $A(\mathrm{H}, \mathrm{Si})$ and $A(\mathrm{H}, \mathrm{C})$. One of them concerns the location of $\mathrm{H}^{-}$. Theory predicts $\mathrm{H}^{-}$to reside in an antibonding $(A B)$ position [33]. So far no experimental evidence exists which could verify these findings. A further question concerns the structure of the ns-states. Are these states also split by the internal stress and by how much? These levels are not accessible with normal optical dipcie transition spectroscopy but may be investigated using two photon spectroscopy.

The same model which has been successfully developed for $A(\mathrm{H}, \mathrm{Si})$ and $A(\mathrm{H}, \mathrm{C})$ applies to $A(\mathrm{Be}, \mathrm{H})$ and $A(\mathrm{Zn}, \mathrm{H})$. The internal stress value $S$, however, has the opposite sign compared to the value for the hydrogen activated centers. Whereas $\mathrm{H}^{-}$activates the neutral impurities $\mathrm{Si}$ and $\mathrm{C}, \mathrm{H}^{+}$partially passivates the double acceptors $\mathrm{Be}$ and $\mathrm{Zn}$. The electronic structures of the resulting complexes are similar insofar as they also have trigonal symmetry and a split $1 \mathrm{~s}$-state. The location of $\mathrm{H}^{+}$may be the bond center $(\mathrm{BC})$ position as it has been firmly established for passivated acceptors in silicon [34]. However, this has not been shown experimentally or theoretically in Ge.

The application of external uniaxial stress to these four hydrogen containing complexes leads to further splitting of the internally split $1 \mathrm{~s}$-state and to the usual splitting pattern in the np-states [35]. The two components of the $1 \mathrm{~s}$-state exhibit orientational splitting for 
stress oriented along [111] and [110] because the trigonal symmetry axes of the various centers assume distinct angles to the stress axis. Stress along [100] affects all centers in the same way and only leads to a shift of the states. Fig. 4 shows the D-and C-lines of the shallow acceptor $\mathrm{Al}$ and the $\mathrm{D}$-line of $A(\mathrm{D}, \mathrm{C})_{2}$ for uniaxial stress applied along [111] and [100]. The subscript 2 identifies all the lines generated by transitions from the ground state component of the split $1 \mathrm{~s}$-state to the $n$ p-states. We will not discuss the splitting pattern of the $\mathrm{Al} \mathrm{C}$-line because the final state leading to the $\mathrm{C}$-line consists of two states which are accidentally degenerate and lead to a very complicated set of lines under stress. Comparison of the $\mathrm{Al} \mathrm{D}$-line and the $A(\mathrm{D}, \mathrm{C})_{2} \mathrm{D}$-line shows the differences in the ground states of these two shallow acceptors. The most obvious difference is seen in the [111] splitting pattern. At $0.039 \mathrm{kbar}$ the Al D-line shows two pairs of lines with very similar strength while the $\mathrm{D}_{1}$ and $\mathrm{D}_{2}$ components of $A(D, C)$ exhibit a line strength ratio of $3: 1$. The Al D-line splits symmetrically in respect to the zero stress position while the $D_{1}$ and $D_{2}$ components are shifted in a 1:3 ratio. The third observation concerns the number of components of the D-lines under stress. There are clearly four Al D-line components while we observe only two components $\mathrm{D}_{1}$ and $\mathrm{D}_{2}$ for $A(\mathrm{D}, \mathrm{C})_{2}$. The stress behavior of $A(\mathrm{H}, \mathrm{Si})$ and $A(\mathrm{H}, \mathrm{C})$ for all three major crystal orientations is fully compatible with the trigonal symmetry complex model with a pre-split $1 \mathrm{~s}$-state.

The optical transition lines of the accepior complexes $A(\mathrm{Be}, \mathrm{H})$ and $A(\mathrm{Zn}, \mathrm{H})$ exhibit similar stress splitting behavior. However, the 3:1 shift of the lines under [111] stress is inverted, indicating an internal stress orientation or level ordering opposite to the orientation of the centers discussed above. Fig. 5 summarizes the splitting patterns of the ground states predicted by the internal stress model for $A(\mathrm{H}, \mathrm{C})$ and $A(\mathrm{Be}, \mathrm{H})$. The internal stress values which lead to the best fit are $\mathrm{S}=-0.810 \mathrm{kbar}$ for $A(\mathrm{H}, \mathrm{C}), \mathrm{S}=-0.438 \mathrm{kbar}$ for $A(\mathrm{H}, \mathrm{Si})$, and $S=+0.205 \mathrm{kbar}$ for $A(\mathrm{Be}, \mathrm{H})$. Using the appropriate values for $\mathrm{S}$ the model quantitatively describes the stress behavior of these complexes with excellent accuracy. 
The inverse ordering of the $l \mathrm{~s}$-states resulting from the opposite signs of $\mathrm{S}$ is reflected in the sequence of the symbols used in the parenthesis of the expression describing each complex, (i.e., $\mathrm{H}$ in first place for $\mathrm{S}<0, \mathrm{H}$ in second place for $\mathrm{S}>0$ ).

A more complete understanding of double acceptor-hydrogen complexes may be reached after the formation of $A(\mathrm{Cd}, \mathrm{H})$ and $A(\mathrm{Hg}, \mathrm{H})$ has been attempted. $\mathrm{Cd}$ and $\mathrm{Hg}$ form well known double acceptors in $\mathrm{Ge}$ and have been used for photoconductor fabrication.

\subsection{The centers $A(\mathrm{Be}, \mathrm{H})$ and $A(\mathrm{Zn}, \mathrm{H})$ in $\mathrm{Si}$}

Single crystal samples of $\mathrm{Si}$ which are doped with the double acceptors $\mathrm{Zn}$ or Be exhibit additional acceptor spectra after exposure to $\mathrm{H}$ at high temperatures. In analogy to the formation of partially passivated double acceptors in $\mathrm{Ge}$, one concludes that the new acceptors are $A(\mathrm{Zn}, \mathrm{H})$ and $A(\mathrm{Be}, \mathrm{H})$.

The spectroscopic signature of the $\mathrm{Zn}-\mathrm{H}$ complex has been reported recently by Merk et al . [36]. They found lines which belong to a hydrogenic acceptor series originating from a ground state at $E_{v}+275.8 \mathrm{meV}$. Substitutic of $H$ with $D$ leads to a shift of $: .49 \mathrm{meV}$ to higher energy. The small isotope shift indicates a static complex. Uniaxial stress experiments will be required to determine the symmetry of the $\mathrm{Zn}-\mathrm{H}$ complex.

The ground state energy of $A(\mathrm{Zn}, \mathrm{H}) \mathrm{E}_{\mathrm{v}}+275.8 \mathrm{meV}$ is nci much shallower than that of $\mathrm{Zn}$ $E_{\mathrm{V}}+319 \mathrm{meV}$ [37]. The relative shift in energy upon partial passivation with hydrogen is much smaller than the shifts observed for the other double acceptor-hydrogen complexes. This difference may be understood in terms of the very large central cell effect which results in a much deeper $\mathrm{Zn}$ ground state than expected from a helium-like impurity with 
two core charges. $\mathrm{H}^{+}$reduces the net-core charge from two to one but does not appear to affect the central cell contribution to the binding energy.

The acceptor $A(\mathrm{Be}, \mathrm{H})$ in $\mathrm{Si}$ has been studied extensively. Crouch $\epsilon t$ al. discovered a new series of acceptor lines in Be doped Si crystals when they added Li [38] or H [39]. They associated the new line series with complexes formed between Be and the interstitial impurities. Muro and Sievers [40] found a large ground state energy shift upon the substitution of $\mathrm{H}$ with $\mathrm{D}$. At very low tempera'ures they found one hydrogenic line series. With increasing temperature a new series originating from t'lermally populated higher $1 \mathrm{~s}$ states appeared. They analyzed the various sets of lines with the tunneling hydrogen model first proposed by Falicov [41]. Peale et al. [42] extended these studies to the 2p' line, a ta ansition from the $1 \mathrm{~s}$ ground state to a hound excited state of the $\mathrm{p}_{1} / 2$ split-off valence band.

They came to the conclusion that the Be-H complex is dynamic with hydrogen either tunneling or undergoing hindered rotor motion. Theoretical calculations find a global total energy minimum for hydrogen residing at a hexagonal interstitial site next to $\mathrm{Be}$ [33]. The energy barrier between neighboring sites is of the order of $0.1 \mathrm{eV}$, compatible with the tunneling model. It should be pointed out that the theoretical calculations give static total energies. In view of the low mass of hydrogen we cannot be certain that the static values describe the system accurately.

\subsection{The acceptor $\mathrm{A}\left(\mathrm{Cu}, \mathrm{H}_{2}\right)$ in $\mathrm{Ge}$}

The most complicated hydrogen related complex which has been studied in detail so far is the triple acceptor $\mathrm{Cu}$ binding two $\mathrm{H}^{+}$. Kahn et al. [43] used PTIS to study five complexes which form between $\mathrm{Cu}$ and $\mathrm{H}, \mathrm{D}$ and $\mathrm{T}$. With the exception of $\mathrm{A}\left(\mathrm{Cu}, \mathrm{H}_{2}\right)$ each complex leads to a single hydrogenic set of optical transition lines. The isotope shifts in the ground state are proportional to the reduced mass of the complex. The acceptor $A\left(C u, H_{2}\right)$, which 
was discovered during the development of ultra-pure germanium [44], has a 1 s-state multiplet with over tien components leading to a very complicazed spectrum. Kahn et al. [43] have explained the difference berween the complex containing two hydrogen ions and all the other combinaltions which contzin at least one heavier isotope with the Devonshire model of the hindered rotor [45]. Growth in a mixed $\mathrm{H}_{2}+\mathrm{D}_{2}$ atmosphere provided unambiguous proof that two hydrogen isotopes are bound to $\mathrm{Cu}$ (see Fig. 1 in [43]).

\subsection{The donor $\mathrm{D}(\mathrm{H}, \mathrm{O})$ in Ge}

The donor $\mathrm{D}(\mathrm{H}, \mathrm{O})$ can be generated in silica crucible and $\mathrm{H}_{2}$ atmosphere grown ultra-pure Ge. During the annealing of the rapid quench acceptor $A(\mathrm{H}, \mathrm{Si})$ the donor $\mathrm{D}(\mathrm{H}, \mathrm{O})$ forms. It has been proposed that after $\mathrm{H}^{-}$breaks loose from $\mathrm{Si}$ at room temperature, it moves through the intrinsic orystal and becomes urapped near oxygen [46]. The porential well of oxygen for $\mathrm{H}$ must be deeper than the well produced by Si, resulting in the experimentally observed higher stability. The $\mathrm{D}(\mathrm{H}, \mathrm{O})$ donor exhibits a number of unique properties which have been studied and analyzed in detail $[3,5,19,31,47,48]$. The extraordinary sharpness of the optical transiticn lines became understood after uniaxial stress measurements showed that the lines did nor split or shift up to high stress values. At a stress of $2.1 \times 10^{7} \mathrm{~Pa}$ in the [111] direction, a new set of lines appears at energies lower by $2.65 \mathrm{meV}$ and the zero stress spectrum rapidly diminishes in intensity. This reduction in intensity can be reversed in par by increasing the sample temperature. This shows that the zero stress ground state continues to exist but it lies above the high stress ground state and can be themally populated. Detailed high resolution studies by Navarro et al. $[49,50]$ show that at least three sets of hydrogenic lines are present. Magnetospectroscopy with alcohol lasers $[18,19]$ led to lines as narrow as $6 \mathrm{keV}$, belonging to the sharpest optical transitions ever recorded for shallow levels in semiconductors. 
Two theoretical models $[47,48,51]$ have been proposed. Both can account for a large fraction of the experimentally observed properties of $D(H, O)$. Space does not permit a detailed discussion of both models and we will only state the major aspects. The tunneling hydrogen model is based on hydrogen rapidly probing four equivalent interstitial sites. The hydrogen motion couples to the donor electron leading to a $\mathrm{i} 6$-fold $1 \mathrm{~s}$ state manifold. Fig. 6 shows the $1 \mathrm{~s}$-state manifold and one of the $n$ p-states. The energy differences have all been derived from experimental observations of the three hydrogenic series $D_{1}, D_{2}$, and $D_{3}$. The fact that transitions from the tighest two-fold degenerate $/ \mathrm{s}$-state have not been observed can easily be explained by the difficulty to thermally populate this state at the temperatures used to perform PTIS.

A static model for $\mathrm{D}(\mathrm{H}, \mathrm{O})$ was first proposed by Broeckx et al. [51] and was later significantly expanded by Ham [48]. This model is based on a static $\mathrm{H}-\mathrm{O}$ complex with trigonal syinmetry. The $1 \mathrm{~s}$ ground state is non-degenerate and is derived from the one conduction band valley which is parallel to the orientation of the H-O complex. As the four conduction band minima shift under stress, the non-degenerate ground states move with them. This explains the stress insensitivity of the $D(H, O)$ lines. At a critical stress in the [111] direction higher lying $I \mathrm{~s}$-state components cross the three states which move up in energy and becorne the new ground states. Ham [48] proposed a number of experimental tests which would permit a decision in favor of the tunneling or the static model.

\section{Summary and Conclusions}

Partially passivated multivalent acceptors and activated neutral impurities offer interesting physics because the bound carrier in the $1 \mathrm{~s}$-state probes the geometry and composition of the complex. PTIS is especially well suited for the study of these centers because it combines high spectral resolution and high sensitivity. Most centers which have been 
studied in Ge and Si are understood rather well, but a number of basic questions remain unanswered. For example, it has not been possible to experimentally determine tile location of hydroger bound to the various impurities. From the experimental and theoretical studies of fully passivated acceptors and donors in Si and GaAs we can conclude that $\mathrm{H}^{+}$occupies high electron density $\mathrm{BC}$ positions while $\mathrm{H}^{-}$prefers $\mathrm{AEl}$ sites. in the absence of large lattice relaxations we may assume that these preferences also apply to partially hydrogen passivated acceptors and hydrogen activated neutral impurities.

The experimental and theoretical possibilities for further productive research appear quite promising. We expect to learn about partially passivated multivalent acceptors and perhaps donors in III-V or II-VI semiconductors. We may ask why partially passivated double donors have not been observed? Do they not form, or have the appropriate conditions not been created? Can the double acceptors $\mathrm{Cd}$ and $\mathrm{Hg}$ in $\mathrm{Ge}$ be partially hydrogen passivated? Many questions remain and there still is much interesting research to be done.

\section{Acknowledgments}

I am indebted to a large number of friends and colleagues with whom I have collaborated and have discussed numerous semiconductor-related topics over the years. I extend my gratitude to them all.

This work was supported in part by the U.S.-National Science Foundation Contract DMR88-06756 and in part by the U.S. Department of Energy under Contract Number DEAC03-76SF00098. 


\section{References}

[1] A. Van Wieringen and N. Warmoltz, Physica 22 (1956) 849.

[2] R.C. Frank and J.E. Thomas, J. Phys. Chem. Solids 16 (1960) 144.

[3] R.N. Hall, Inst. Phys. Confr. Ser. 23 (1975) 190.

[4] W.L. Hansen and E.E. Haller, Mat. Res. Soc. Proc. Vol. 16 (1983) 1.

[ 5] E.E. Haller, W.L. Hansen, and F.S. Goulding, Adv. in Phys. 30, No. 1 (1981) 93.

[6] C.T. Sah, J.V.C. Sun, and J.J.T. Tzou, Appl. Phys. Lett. 43 (1983) 204; J. Appl. Phys. 54 (1983) 4378.

[7] J.I. Pankove, D.E. Carlson, J.E. Berkeyheiser, and R.O. Wance, Phys. Rev. Lett. 51 (1983) 2224.

[8] J.I. Pankove, R.O. Wance, and J.E. Berkeyheiser, Appl. Phys. Lett. 45 (198 1100.

[9] R.N. Hall, in: Proc. Semiconductor Materials for Gamma Ray Detectors, W.L. Brown and S. Wagner, eds. (1966) p. 27.

[10] J. Czochralski, Z. Phys. Chemie 92 (1918) 219.

[11] W.L. Hansen, E.E. Haller, and P. Luke, IEEE Trans. Nucl. Sci. NS-29, No. I (1982) 738.

[12] E.E. Haller, W.L. Hansen, P. Luke, P. McMurray, Jr., and B. Jarett, IEEE Trans. Nucl. Sci. NS-19, No. 1 (1982) 745.

[13] J.H. Yee, S.P. Swierkowski, G.A. Armantrout, and R. Wichner, J. Appl. Phys. 45 (1974) 3949.

[14] See for example Fig. 2 in Ref. 5.

[15] T.M. Lifshits and F. Ya Nad, Soviet Phys. Dokl. 10 (965) 532; for a detailed review see: Sh. M. Kogan and T.M. Lifshits, Phys. Stat. 'Sol. (a) 39 (1977) 11.

[16] R.J. Bell, in: Introductory Fourier Transform Spectroscopy (Academic Press, N.Y., 1972). 
[17] T. Theiler, F. Keilmann and E.E. Haller, Inst. Phys. Confr. Ser. 95 (1988) 179.

[18] E.E. Haller, H. Navarro and F. Keilmann, in: Proc. 18th Int. Confr. Phys. Semic., O. Engstrom, ed. (World Sci. Publ., Singapore, 1987) p. 837.

[19] H. Navarro, E.E. Haller, and F. Keilmann, Phys. Rev. B 37 (1988) 10822.

[20] N. Nishikawa and R. Barrie, Can. J. Phys. 41 (1963) 1135.

[21] R. Barrie and K. Nishikawa, Can. J. Phys. 41 (1963) 1823.

[22] M.S. Skolnick, L. Eaves, R.A. Stradling, J.C. Portal, and S. Ask Comm. 15 (1974) 1403.

[23] L. Darken and S.A. Hyder, Appl. Phys. Lett. 42 (1983) 73í.

['24] M.J.H. van de Steeg, H.W.H.M. Jongbloets, J.W. Gerritsen and P. Wyder, J. Appl. Phys. 54 (1983) 3464.

[25] M.J.H. van de Steeg, H.W.H.M. Jongbloets, and P. Wyder, J. Appl. Phys. 50 (1983) 1234 and Phys. Rev. B 30 (1984) 3374.

[26] J.A. Wolk, E.E. Haller, D.D. Nolte, R.E. McMurray, Jr., E. Bauser, and I. Silier, Bull. Am. Phys. Soc., Series II, 35, No. 3 (1990) 413.

[27] D. Larsen, Phys. Rev. B 13 (1976) 1681.

[28] E. Janzen, G. Grossmann, R. Stedman, and H.G. Grimmeiss, Phys. Rev. B 31 (1985) 8000; see also Phys. Rev. B 29 (1984) 1907.

[29] M. Kleverman, J. Olajos, and H.G. Grimmeiss, Phys. Rev. B 37 (1988) 2613.

[30] H.G. Grimmeiss, M. Kleverman, and J. Olajos, Mat. Res. Soc. Proc. Vol. 163 (1990) 3.

[31] E.E. Haller, Phys. Rev. Lett. 40 (1978) 584.

[32] J.M. Kahn, R.E. McMurray, Jr., E.E. Haller, and L.M. Falicov, Phys. Rev. B 36 (1987) 8001 .

[33] P.J.H. Denteneer, C.G. Van de Walle, and S.T. Pantelides, Phys. Rev. Lett. 62 (1989) 1884. 
[34] K. Bergman, M. Stavola, S.J. Pearton, anc' T. Hayes, Phys. Rev. B 38 (1988) 9643.

[35] A.K. Ramúas and S. Rodrigues, Repts. Progr. Phys. 44 (1981) 1297.

[36] E. Merk. J. Heyman, and E.E. Haller, Mat. Res. Soc. Proc. Vol. 163 (1990) 15.

[37] E. Merk. J. Heyman, and E.E. Haller, Sol. State Comm. 72 (1989) 851.

[38] R.K. Crouch, J.B. Robertson, and T.E. Gilmer, Jr., Phys. Rev. B 5 (1972) 3111.

[39] R.K. Crouch, J.B. Robertson, H.T. Morgan, T.E. Gilmer, Jr., and R.K. Franks, J. Phys. Chem. Solids 35 (1974) 833.

[40] K. Muro and A.J. Sievers, Phys. Rev. Lett. 5' (1986) 897.

[41] E.E. Haller, B. Ioós, and L.M. Falicov, Phys. Rev. B 21 (19⿺辶0) 4729.

[42] R.E. Peale, K. Muro, and A.J. Sievers, Phys. Rev. B 411 (1990) 588.

[43] J.M. Kahn, L.M. Falicov, and E.E. Haller, Phys. Rev. Lett. 57 (1986) 2.077.

[44] E.E. Haller, G.S. Hubbard, and W.L. Hansen, IEEE Trans. Nucl. Sci. NS-24, No. 1 (1977) 48.

[45] A.F. Devonshire, Proc. R. Soc. London Ser. A 153 (1936) 601.

[46] E.E. Haller, Physica 146B (1987) 201.

[47] B. Joós, E.E. Haller, and L.M. Falicov, Phys. Rev. B 22 (1980) 832.

[48] F.S. Ham, Phys. Rev. B 38 (1988) 5474.

[49] H. Navarro, J. Griffin, and E.E. Haller, J. Phys. C: Sol. State Phys. 21 (1987) 1511 .

[50] H. Navarro, J. Griffin, E.E. Haller, and R.E. McMurray, Jr., Sol. State Comm. 64 (1987) 1297.

[51] J. Broeckx, P. Clauws, and J. Vennik, J. Phys. C 13 (1980) L141. 


\section{Figure Captions}

Fig. 1 Schematic illustration of the two-step excitation process of PTIS. The energy between the ground state and the first excited state is not shown to scale. It is much larger than the energies between the excited states.

Fig. 2 Evolution of the PTIS line spectrum with increasing temperature. At $\mathrm{T}=0$ only the photoconductive continuum exists because no photons are present to provide the thermal excitation from a bound excited state to the band.

Fig. 3 PTI spectrum of $p$-type ultra-pure Ge without a) and with b; band edge illumination. The original labeling in Ref. 22 has been updated. The line series belonging to a donor $\mathrm{S}$ is úe to the donor complex $\mathrm{D}(\mathrm{Li}, \mathrm{O})$. The donor $\mathrm{X}$ is the hydrogen containing donor complex $\mathrm{D}(\mathrm{H}, \mathrm{O})$.

Fig. 4 PTI spectra of the D- and C-transitions of Al, and the D-transition of $A(D, C)_{2}$, for uniaxial stress applied along [111] and [100]. All spectra were recorded at $7.0 \mathrm{~K}$.

Fig. 5 The piezospectroscopic behavior of the two (ls)-like levels of differently oriented, trigonal shallow acceptor complexes, based on the "equivalent stress" model. (a) Trigonal distortion equivalent to a stress of $+0.205 \mathrm{kbar}$ (tensional); (compressional). Roman numerals denote the four possible orientations of the complexes. " $\Lambda_{4}$ " and " $\Lambda_{5,6}$ " denote the representations of $C_{3 v}$ according to which the states transform, in the absence of externally applied stress. The energy shifts are shown for externally applied compressional stress. (From Ref. 32)

Fig. 6 The $1 \mathrm{~s}$-state manifold and one of the $n$ p-states of the $\mathrm{D}(\mathrm{H}, \mathrm{O})$ donor according to the $\mathrm{H}$ tunneling model. (From Ref. 50) 


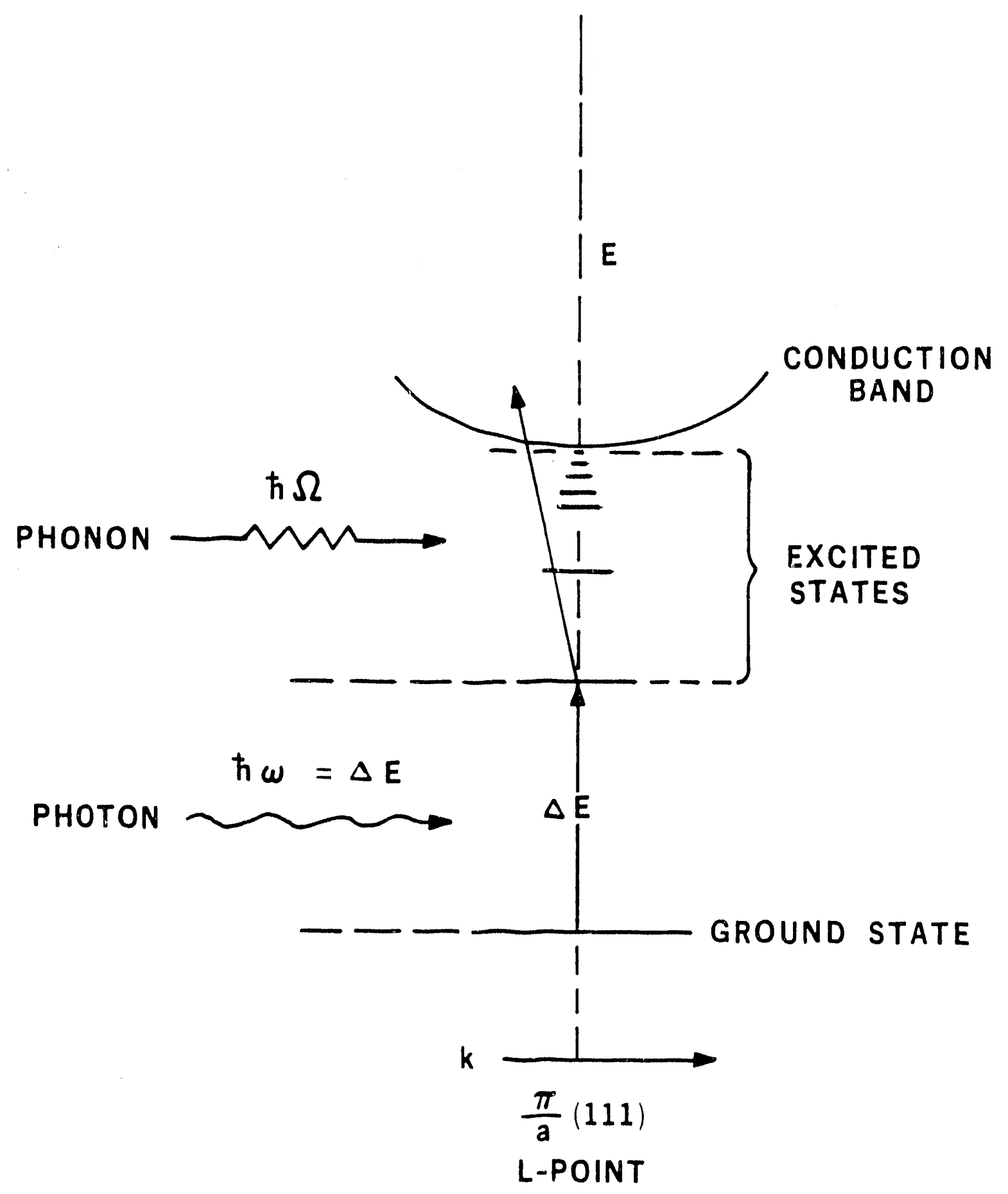

XBL 7411.8629

Figure 1. 


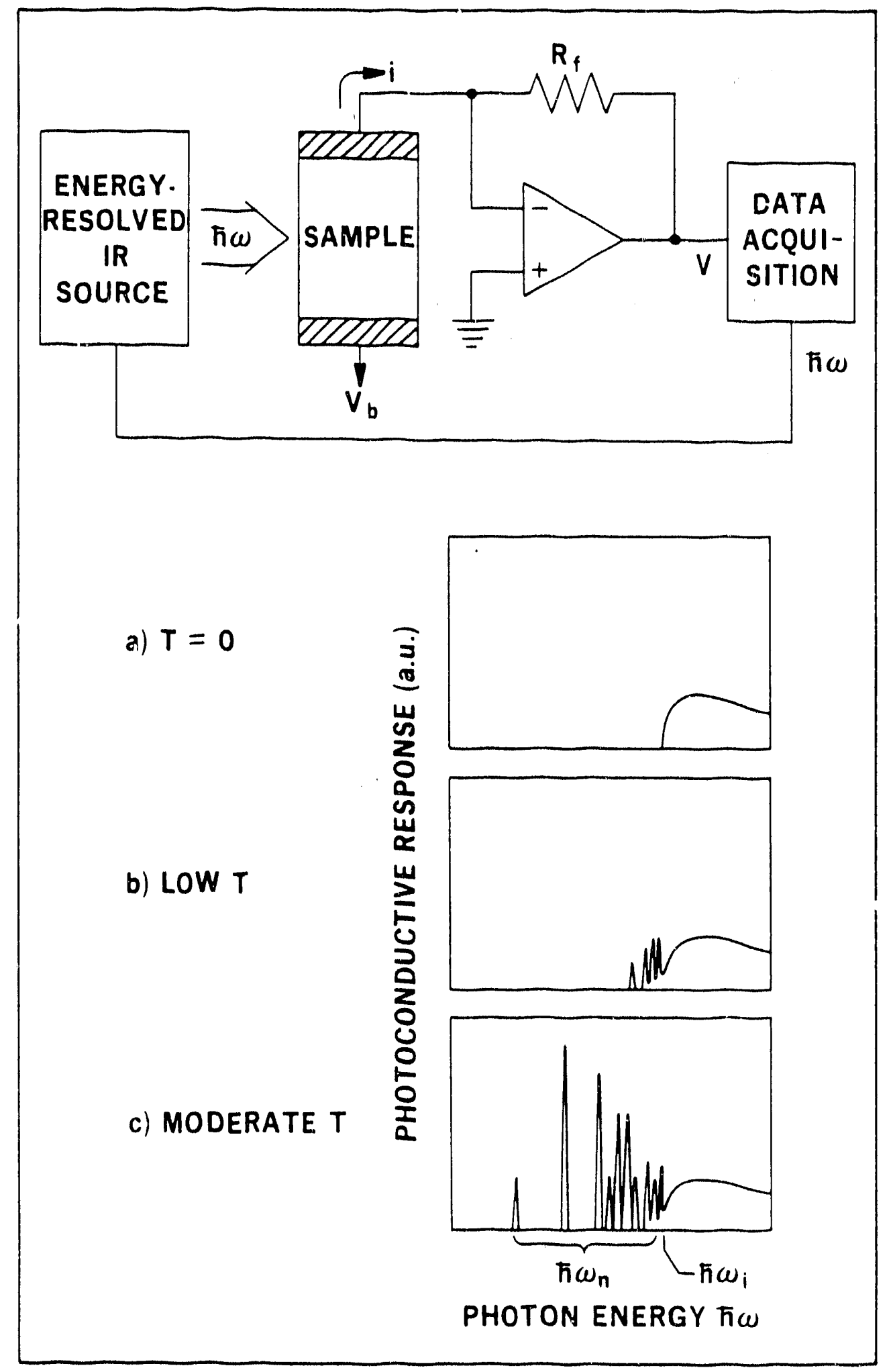

XBL $8610-3834$

Figure 2. 


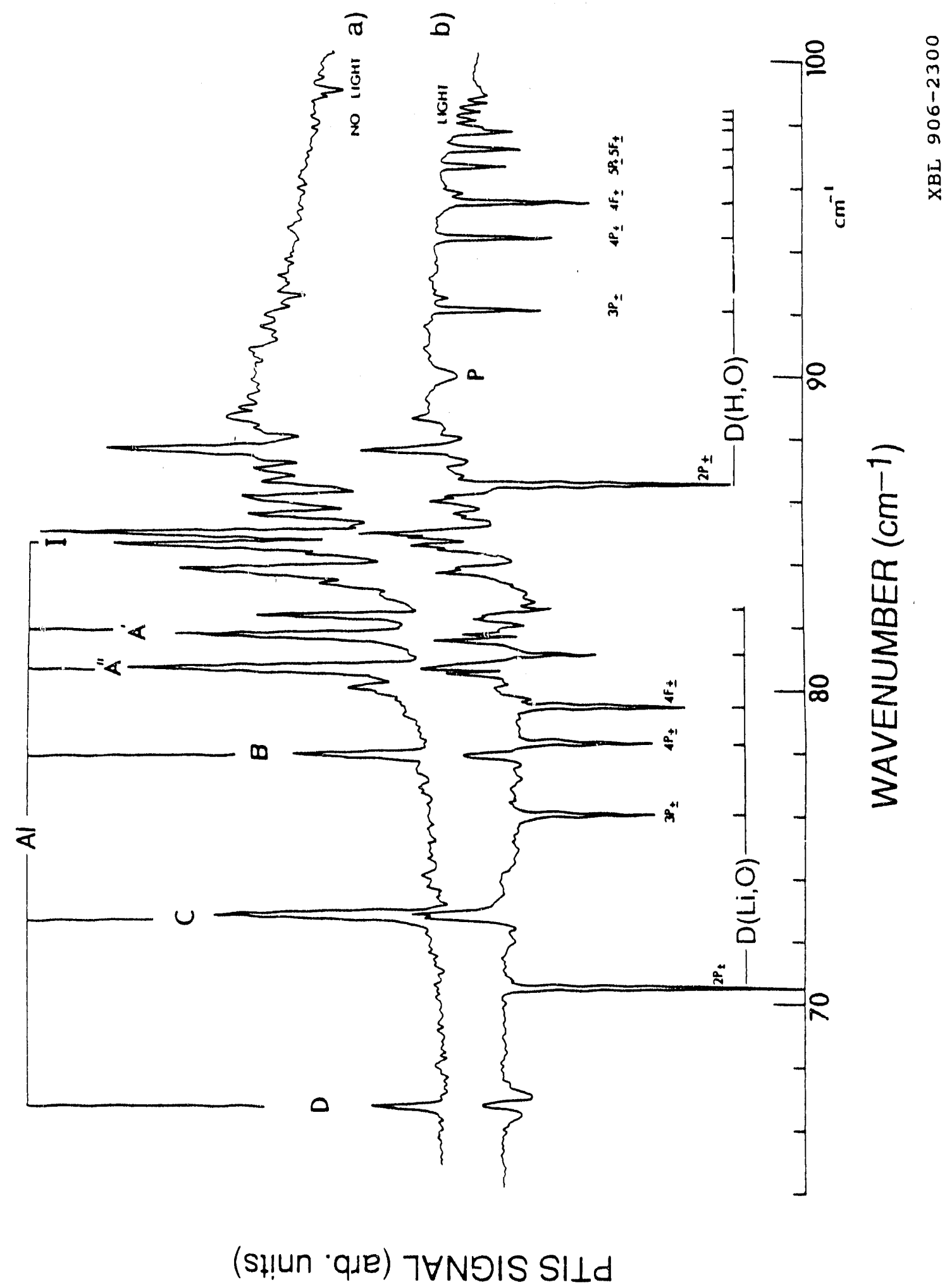

Figure 3. 


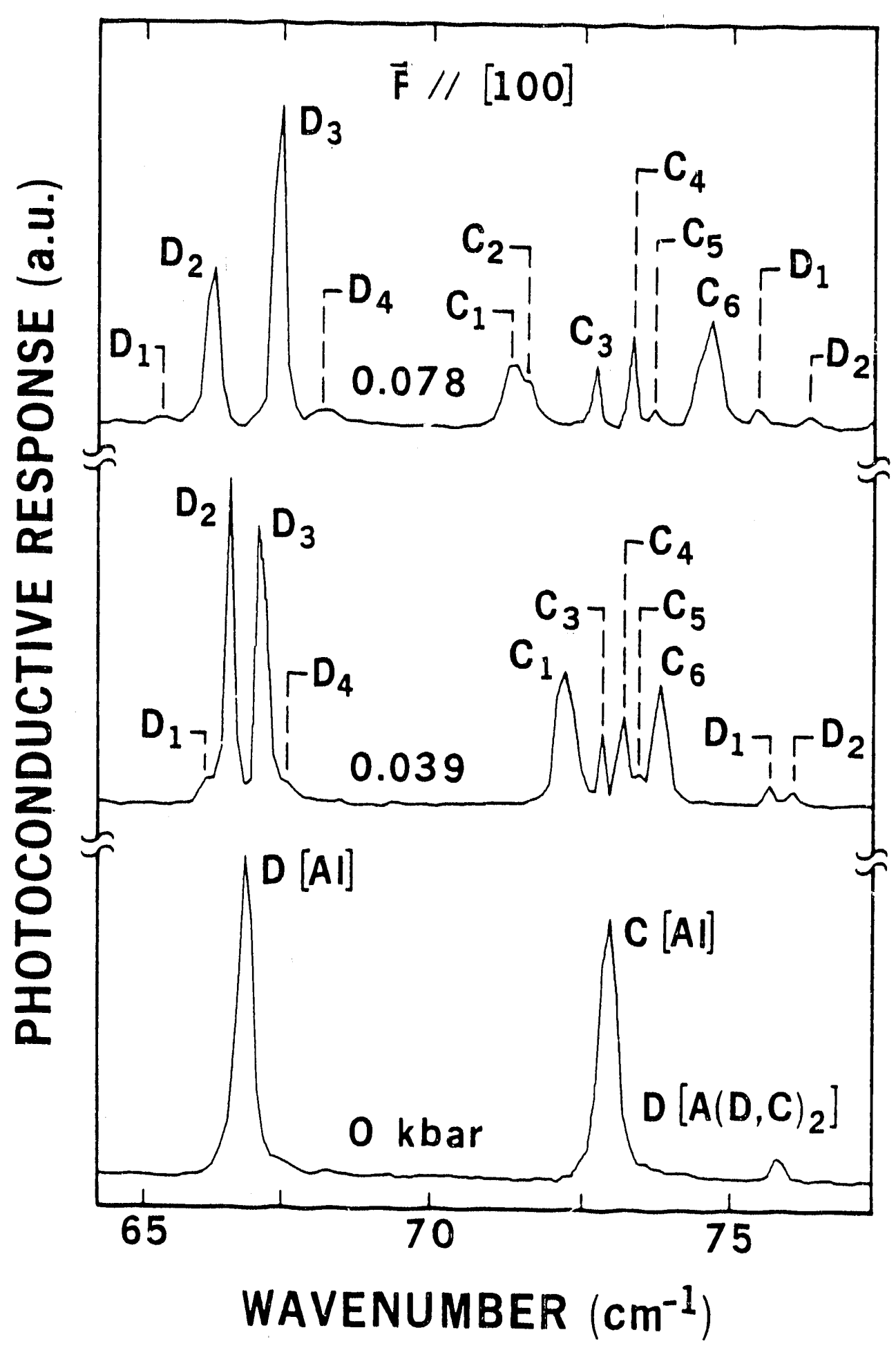

XBL 8611-4389

Figure 4.

(right half) 


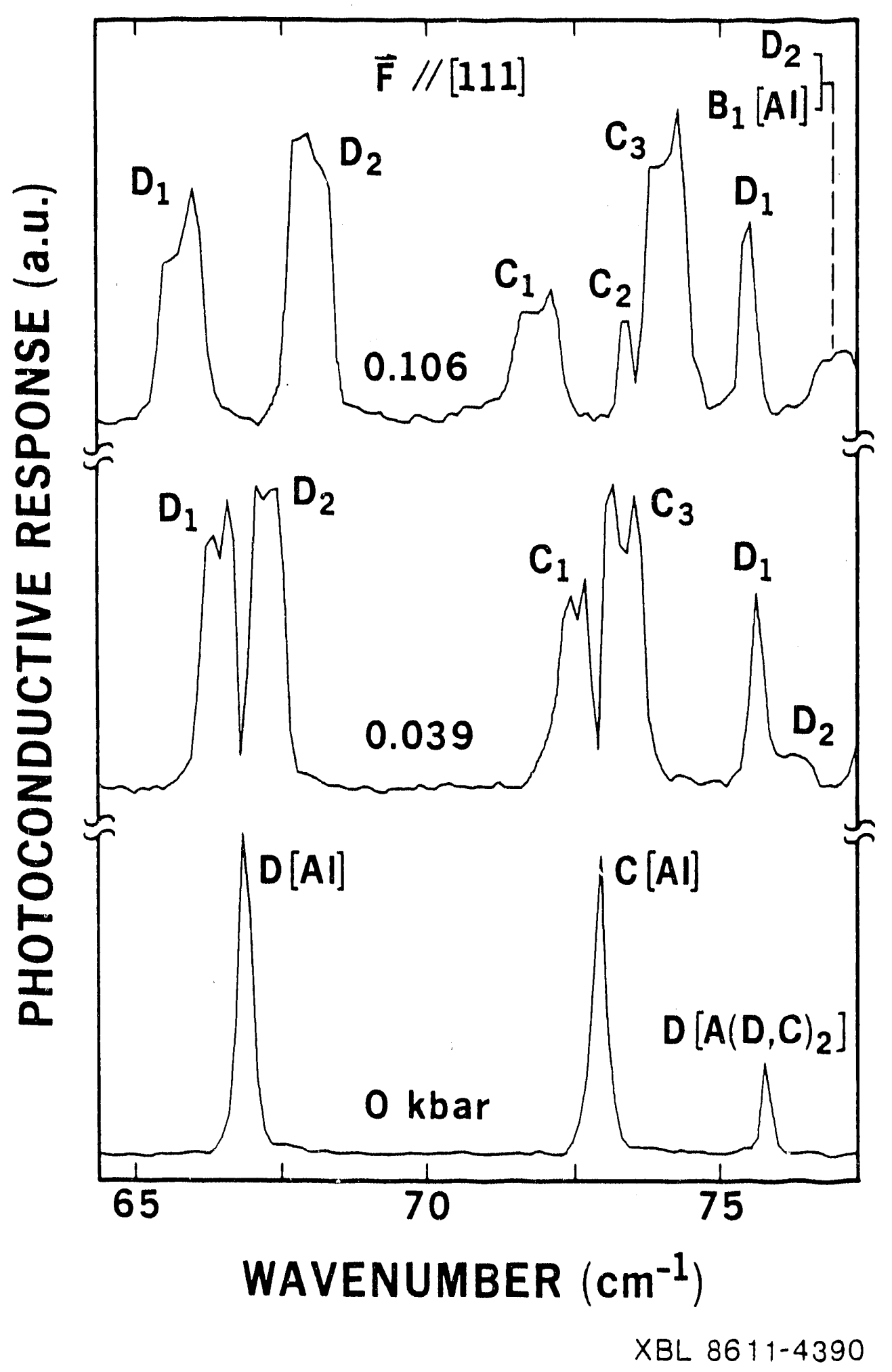

Figure 4.

(left half) 

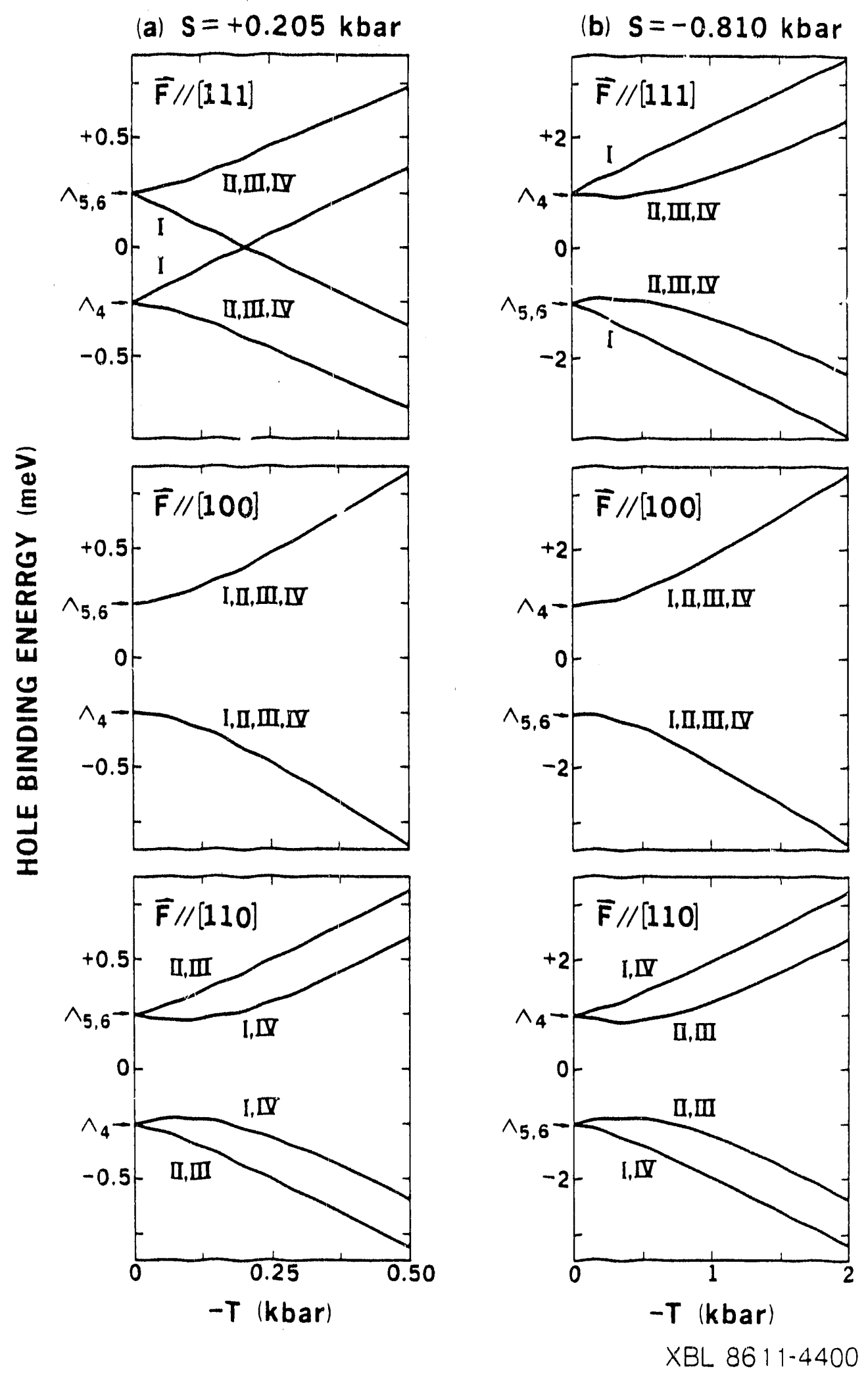

Figure 5. 


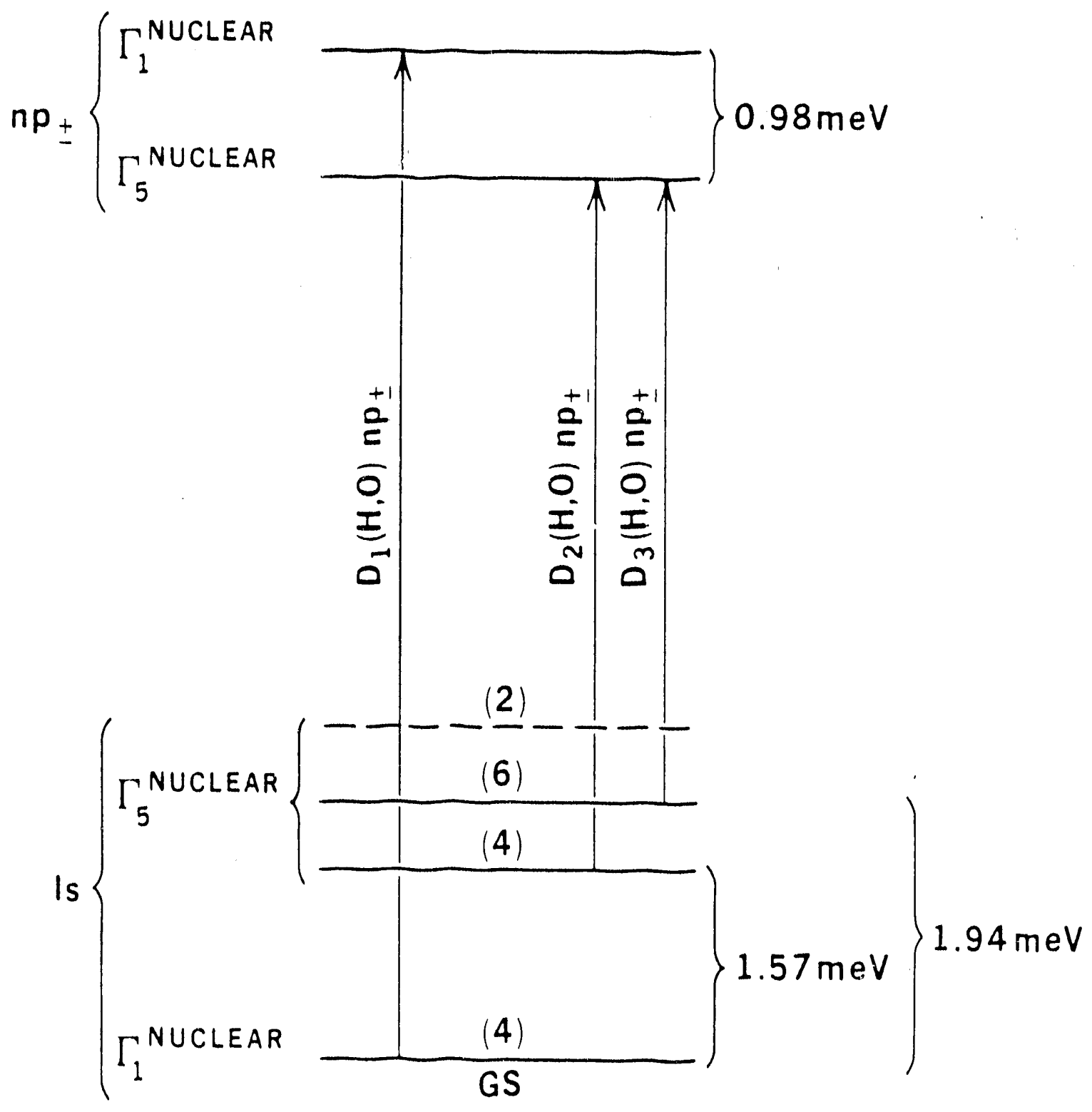

XBL $87+-1501$

Figure 6. 

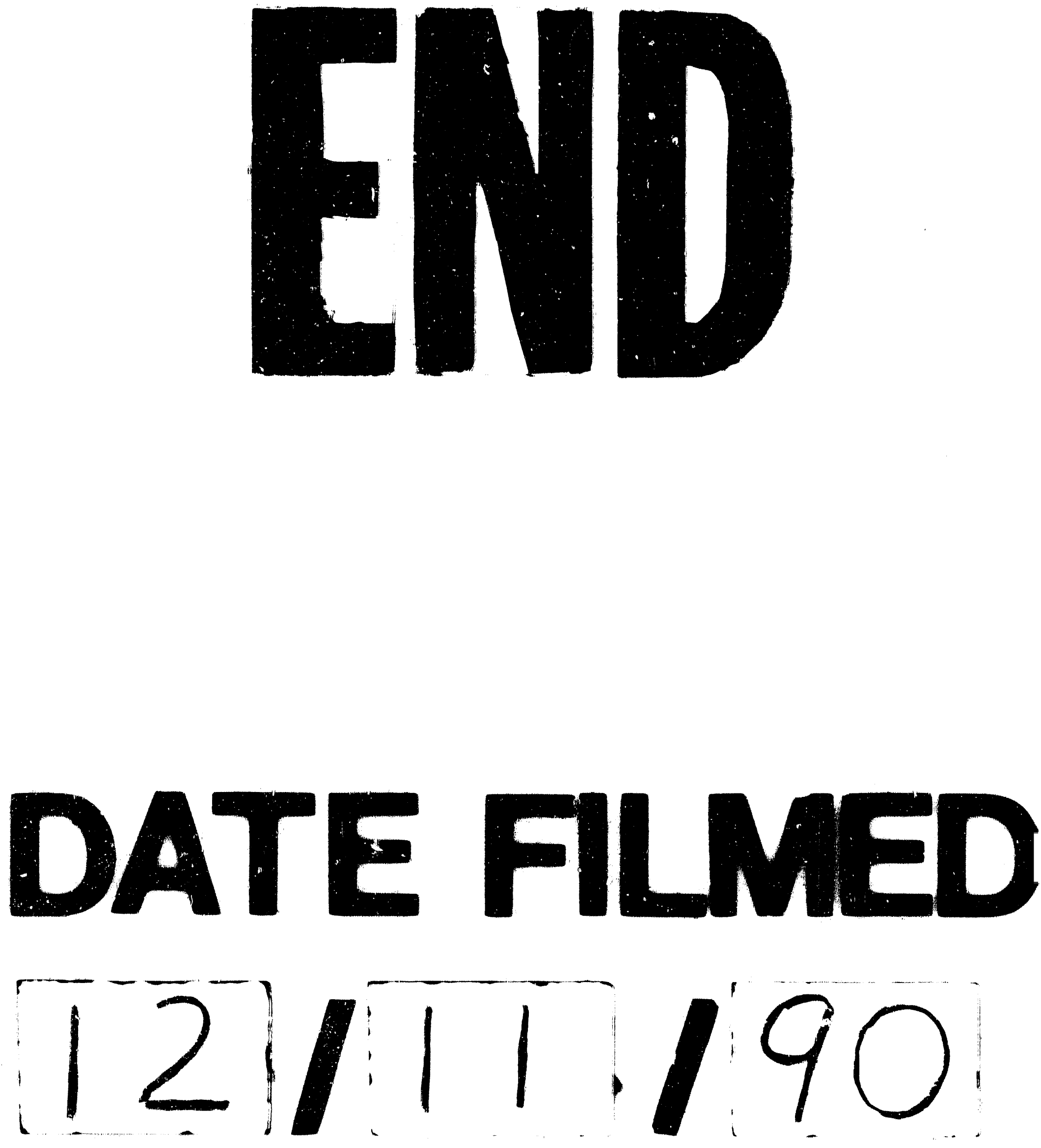
